\title{
A Comparative Study on Harvesting Plan Predicting Insurance with Two-Stage Stochastic Analysis
}

\author{
Hashnayne Ahmed*, Shek Ahmed \\ Department of Mathematics, Faculty of Science and Engineering, University of Barishal, Barishal, Bangladesh \\ Email address: \\ hashnayneahmed17@gmail.com (H. Ahmed),shekmathdu@gmail.com (S. Ahmed) \\ ${ }^{*}$ Corresponding author
}

To cite this article:

Hashnayne Ahmed, Shek Ahmed. A Comparative Study on Harvesting Plan Predicting Insurance with Two-Stage Stochastic Analysis. International Journal on Data Science and Technology. Vol. 5, No. 4, 2019, pp. 73-82. doi: 10.11648/j.ijdst.20190504.12

Received: December 9, 2019; Accepted: December 20, 2019; Published: December 31, 2019

\begin{abstract}
The exception of considering uncertainty could be very detrimental to the outcomes of any systems or phenomena in the long run. Stochastic Process describes the way of considering uncertainty in different sectors of our life. We use Linear Programming for planning at its best. It is also considered as the best optimization technique for taking decisions or planning. But this planning tool disappoints us in optimization for unexpected risk or stochasticity. Consideration of stochasticity for a farmer to devote land on different crops for harvesting could be some insurance for the farmer with the best possible outcomes. Stochastic Programming studies these types of optimization techniques with risk consideration for better decisions in every step of our life. In this paper, we described the early starting of uncertainty calculation or stochastic approach and the evolution of stochastic optimization fields. Stochastic optimization is rather important in the sense of uncertainty calculation than sensitivity analysis and works through data gained from experience. We also present a stochastic model with some uncertainty issues in harvesting to make better outcomes. Some application areas are also discussed.
\end{abstract}

Keywords: Stochastic Optimization, Stochastic Programming, Stochastic Modeling, Uncertainty Calculation, LINDO

\section{Introduction}

We want the best output in every step of our life with the limited resources we hold. That's why it is necessary for us that we make the best decision in every case. A good decision can help us to get a better output. A study that assists to choose our decision for the best possible outcomes is called optimization techniques. The optimization problem grows everywhere as we always seek the best output. Stochastic Process is one of the most used techniques for optimization. It is widely used in Mathematics, Finance, Economics, Medical Science, Engineering fields, etc. Stochastic Process discusses the matters where risk arises. And in the world risk is everywhere, nothing is certain. [8]

Stochastic programming is the study of decision making under risk management in Optimization techniques. It was first introduced by George B. Dantzig by considering some cases related to uncertainty. We face uncertainty in every section of our life. Our expectation hampers in most cases due to uncertain parameters. These days harvesting is more challenging due to uncertain parameters arises in harvesting. A farmer has to make the decision on devoting lands for crops with a better outcome expectation. But in most cases, they fail to make a better decision due to uncertain parameters like a weather forecast, productivity of lands, selling price fluctuations, etc. Stochastic Programming could be an asset by calculating the uncertain parameters for the most possible better output.

There are many problems due to uncertainty. Influence maximization in social networks [15], Maintenance and Rehabilitation planting of Pavements [16], Ecological water requirements [17], Graph Routing Problem with risks [4, 22], supply chain networks [18] are some examples where stochastic optimization techniques could be applied. Based on the problems arises for the consideration of a variety of risks in different periods of a system or phenomena, Stochastic Programming could be sectorized. The consideration of risks or uncertainty could help us to make better decisions in every step of our life. 


\section{Meaning of Stochastic}

The word 'Stochastic' is a Greek word meaning something like 'aim' or 'goal'. But the word 'stochastic', we are familiar with means something happened randomly or some random approaches, which is a German word. This word appeared in English to describe some mathematical things known as the Stochastic Process. But stochastic refers to something random and so 'Stochastic Process' may refer to some randomly determined process. That is, we call it a random process for the stochastic process. $[10,11]$

For example, let one have to go from the place ' $\mathrm{P}$ ' to ' $\mathrm{Q}$ ' in the following figure. There are three different ways to go to the destination. He can randomly choose any of the three ways.

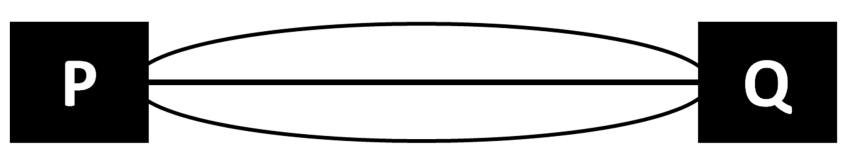

Figure 1. Randomness.

Similarly, we can randomly pick some students from a class, randomly choose lottery tickets and many more. Anything could happen in the future, that is also something random. That is anything that is not fixed is something random, could be happening or couldn't. We may also call it something uncertain.

\section{Stochastic Process}

\subsection{Background}

We live in an uncertain world. Everything around us is uncertain, nothing is certain. We don't know what happened next. A farmer could not know about the future, what will be the weather conditions in the next future. A fisherman doesn't know how many fish he will catch. A common man doesn't know whether this day will be good or bad for him. Even a researcher doesn't know about the result of this future experiments. Everywhere what we do is a prediction, predict based on the previous data. Most time these predictions result well. But is the data available everywhere, in every case? What will happen if a farmer needs to know about the weather conditions about six months earlier? What will be the predictions for share-markets? What will be the most predictable one chance?

Random things occurred in our world. Everything is uncertain or risks everywhere, anything could happen in our daily life means stochastics is everywhere. The study calculates these random things and brings us the most possible assumptions is called Stochastic Process. It is not obvious that it will be always something beneficial by calculating stochastically rather it may indicate the middle way. In case of business, something that will give you a better profit considering your losses or risks due to some uncertain cases like weather, death, etc. or some random process deficit.

\subsection{Uncertainty and Stochasticity}

In Stochastic Programming, three words are mostly used to describe stochasticity: uncertainty, randomness, and risk. Something is uncertain if you don't know the exact thing from many things. But if you can specify the probability of the exact thing among many things, it is stochastic then. Stochasticity means randomness, not exactly uncertainty. It means uncertainty with a possible probability distribution. For example, the weight of a book is uncertain even after you pick it up. Here the mass of the book is not random or not changes randomly and that's why it is not stochastic. Again, if we toss a coin, we know the possible probability distribution of the output and it means a coin toss is stochastic. It is easy to observe that, where is uncertainty is risks.

Remark 3.2.1. If the probability of a stochastic event is one, then the outcome is certain.

\subsection{Stochastic Approach}

A collection of random variables that are indexed by some mathematical set where each random variable is connected with an element in the set is called the Stochastic Process or Random Process. If we denote a set of time-indexed random variables by $X_{t}$, then the stochastic Process is the family of the random variables defined as,

$$
\left\{X_{t}: t \in I\right\}
$$

where index set is $I$ which is a subset of the set of all real numbers. [9, 10]

Aleksandr Khinchin, a soviet mathematician gave the first mathematical definition of a Stochastic Process as a family of random variables indexed by the real line in the early 1930s. Other mathematicians as Andrey Kolmogorov, Joshep Doob, William Feller, Maurice Rene Frechet, Paul Levy, Wolfgang Doeblin, Harald Cremer also put considerable effort in the establishment of Stochastic Process. [11]

To construct a Stochastic Process, there are two main approaches: Considering a space of functions defining a probability distribution to each element of the function or considering a set of random variables. Stochastic Process is an active topic in Mathematics, especially in Probability Theory for research as it is important for both theoretical reason and application. The stochastic Process is broadly used in mathematical models of systems and phenomena's that occur randomly.

\section{Stochastic and Deterministic World}

The theory of Stochastic Process is a field of Mathematics which deals with probabilistic systems evolving over time. The simplest Probabilistic system appears when you toss a coin. Tossing a coin is widely used in various games to determine the first. Here it is just a mechanical action, simply accelerate the coin and it moves according to some physical laws like gravity, air resistance, etc. Let's forget the physical background and concentrate on the result of the experiment, 
it can be either head or tail. That is, for both outcomes the probability is one-half for each. But actually, in some sense, you moved from deterministic (gravity, air resistance) to stochastic (randomness) world. [10]

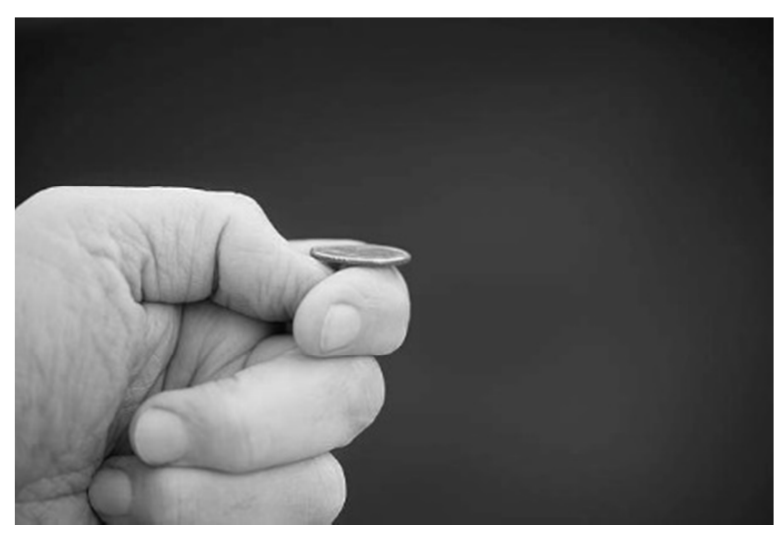

Figure 2. Tossing a coin.

\section{Linear Programming}

The Mathematical Technique for optimizing a linear function of several variables (maximizing output or minimizing cost) is called Linear Programming. It can also be called linear optimization. It is a process to gain optimizing output (maximizing output or minimizing cost) as required whose conditions are represented with the problem by using linear relationships. The linear function which has to be optimized is called the objective function and all the conditions expressed by linear relationships are called the constraints of the linear programming problem.

A linear programming problem can be expressed as the following canonical form:

Optimize (Maximize or Minimize) $z=c^{T} x$

Subject to $A x(\geq$,or $=$, or $\leq) b$

And $x \geq 0$

where the vector of variables is $x$ and the vectors of the coefficient function are $c, b$. [20]

Remarks 5.1. Sometimes 'Max' is written for Maximize and 'Min' is written for Minimize.

\section{Stochastic Programming}

\subsection{Why Stochastic Programming}

Mathematical Programming (Optimization) is about decision making or planning. Stochastic Programming is about decision making under uncertainty. We may also call it "Mathematical Programming with random parameters."

In most applications of optimization, randomness is ignored. Otherwise, it is dealt with using Sensitivity analysis. But For large-scale problems, sensitivity analysis is useless. No matter how careful you are, you can't get rid of inherent randomness. Stochastic Programming is the way.

\subsection{History of Stochastic Programming}

The first programming formulation of a problem and a solving process for it was given by Soviet economist Leonid Kantorovich in 1939, which is the foundation of Linear Programming (LP). Then in 1946, General Linear Programming formulation was developed by George B. Dantzig. It was about planning problems in the US air force. To solve the formulated Linear Programming Problem (LPP), Dantzig also proposed the well-known Simplex Method. Linear Programming focused on optimization of the linear objective function with respect to some requirements. In Linear Programming, we assume all the parameters of the problem are known numbers. [2, 3]

Most Practical decision problems involve uncertainty. Parameters are uncertain because of the lack of reliable data, measurable errors, future, and unobservable events. For the uncertainty or risks, the formulation of a developed framework that calculates the risk and gives the wished optimization with some unknown parameters is called the Stochastic Programming. Stochastic Programming stands on Robust Optimization Techniques [1950], where the parameters are known within certain bounds. [3, 13]

The beginning of Stochastic Programming (SP) or in particular Stochastic Linear Programming (SLP), dates back to the '50s and early '60s of the last century. In 1955, George B. Dantzig formulated the general formulation of linear programming with uncertain data. A. Charnes and W. W. Cooper introduced a particular stochastic program with chance constraints in 1959. In 1961, Beale proposed a quadratic programming approach to solve special simple recourse stochastic programs. Besides A. Madansky, G. Tintner, John R. Birge, Francois Louveaux, C. van de Panne, W. Popp, H. Brunn, H. Minkowski, A. Prekopa, J. F. Benders, G. Zoutendijk played the significant roles behind the establishment of today's Stochastic Programming. [2, 3, 13]

Stochastic programming takes advantage of the probability distributions, where the rest is as same as Robust Optimization. Finding some policy that is feasible for all possible data to optimize the objective function (decision and random variables) is the aim of Stochastic Programming.

\subsection{Data Requirements for Stochastic Programming}

Data requirements for Stochastic Programming models are similar to the Linear Programming models except for randomness consideration. The addition data requirements in Stochastic Programming are representing uncertainty with some probability distributions. In most cases for stochastic programming, it is easy to consider some scenario distribution based on experience. A model with some random variables is easier to represent for computational algorithms. $[6,7]$

\subsection{Introductory Stochastic Programming}

There is a French saying, "The art of managing is anticipating."

It is obvious that the future cannot be perfectly foreknew rather it should be considered as random or uncertain. The aim of Stochastic Programming is finding an optimal 
decision with random data. In that sense, stochastic is opposed to deterministic. [1]

A Stochastic Programming problem can be expressed as the following canonical form:

Optimize (Maximize or Minimize) $z=c^{T} x+E_{\xi} Q(x, \xi)$

Subject to $A x(\geq$ or $=$, or $\leq) b$

And $x \geq 0$

where the vector of variables is $x$ and the vectors of the coefficient function are $c, b$. The second term in the objective function comes for uncertain data. $E_{\xi}$ is referred to as the Mathematical Expectations with respect to some random vector $\xi$. [1, 5, 12]

Stochastic Programming is decision making under risks. It may also refer to optimization under uncertainty. The field of Stochastic Programming is enriching with apace as contributions from many disciplines such as mathematics, finance, economics, operations research, probability, and statistics. [3]

\section{Classifications of Stochastic Programming}

There are many kinds of Stochastic Programming. Some mostly used kinds are: [1]

a) Two-Stage Stochastic Linear Programs with Fixed Recourse

The decision-maker takes some decision considering the randomness of some event or phenomena in the first stage. After that, some random event occurs and affects the outcome of the first stage decisions. The decision-maker has to make some second stage or recourse decision based on the outcome which may defend any bad effects that might have been experienced.

\section{b) Probabilistic or Chance Constraints}

It considers the term uncertainty of mother nature with many scenarios for taking the first stage decision which results from a big number of extra constraints including chance or probabilistic constraints.

c) Stochastic Integer Programs

It simply requires some integer in the first stage or in the second stage or both decisions while the Linear Programming problem deals with the variables that are non-negative real numbers.

\section{d) Multi-stage Stochastic Programs with Recourse}

For making the best decision on a system or phenomenon, more stages are needed. Multi-stage stochastic programs consist of a sequence of decisions that affect the outcomes over a time period.

e) Stochastic Non-linear Programs with Recourse

In this case, the objective function occurred in non-linear form like stochastic approaches for quadratic programming, quasi-concave programming, etc.

\section{Model Analysis}

\subsection{A Farmer's Scenario}

Let's introduce a local Bangladeshi farmer Abdur Razzak, who lives with his two sons near Karapur village (along Nobogram Road) at Barishal District, Bangladesh. He has a total amount of 1 -acre $(43,560$ square feet or about $2.52 \mathrm{Kani}$, a local land measurement unit) cornfield. He specializes in harvesting rice (irrigation), potatoes, chilies, groundnuts and pumpkins during the winter season in Bangladesh (December, January, February). We want to help the farmer for deciding how much land he should devote to each crop with the most possible benefits.

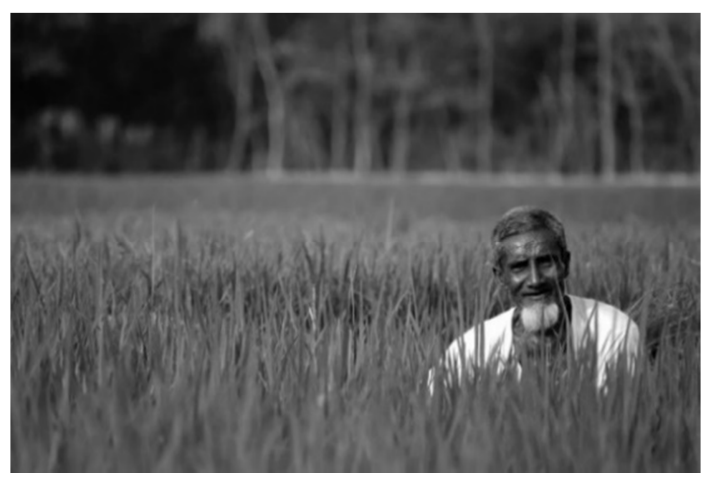

Figure 3. Abdur Razzak.

He also raises 4 cows for producing milk or meat as per his choice. He needs rice and potato plants for the cattle feeds during that time. Since potato plants are available after cropping potatoes so it has no effect on production but he needs 0.25 metric ton potatoes for a home consumes. He needs rice plants before cropping paddy and that costs him about 0.5 metric tons of rice for cattle feed and household consumes, needed to raise in his lands. (1 metric ton equals 1000 kilograms)

According to his experience, he knows that the mean yield per 0.4 acres ( $1 \mathrm{Kani}$ ) is 0.8 metric tons for rice, 3 metric tons for potatoes, 0.12 metric tons for chilies, 0.2 metric tons for groundnuts and 2.5 metric ton for pumpkins. Another profitable process is cultivating pumpkins with potatoes (mixture) which yields a total amount of 2.5 metric tons of potatoes and 1 metric ton pumpkins per 0.4 -acre land. The farmer prefers this mixture process as it utilizes the land properly. The planting cost per 0.4 -acre land is 24000 Taka for rice, 9600 Taka for chilies, 12000 Taka for groundnuts, 28000 Taka for the mixture of potatoes and pumpkins.

The average selling price (since it varies) for the crops are given below:

Table 1. Selling Prices.

\begin{tabular}{llllll}
\hline Crops & Rice & Potatoes & Chilies & Groundnuts & Pumpkins \\
\hline Selling Price (Taka/Metric Ton) & 32,000 & 7,000 & 100,000 & 60,000 & 20,000 \\
\hline
\end{tabular}




\subsection{Formulation and Estimation}

To help the farmer on deciding how much land he should devote to each crop, let's take some variables for making a programming model:

$x_{1}=$ Amount of land assigns for rice (acres),

$x_{2}=$ Amount of land assigns for potatoes and pumpkins (acres),

$x_{3}=$ Amount of land assigns for chilies (acres),

$x_{4}=$ Amount of land assigns for groundnuts (acres), $y_{1}=$ Amount of rice sold (metric tons),

$y_{2}=$ Amount of potatoes sold (metric tons),

$y_{3}=$ Amount of chilies sold (metric tons),

$y_{4}=$ Amount of groundnuts sold (metric tons),

$y_{5}=$ Amount of pumpkins sold (metric tons),

For constructing the above problem, let us first convert the given data for per-acre land as follows:

Table 2. Data for the problem.

\begin{tabular}{lllll}
\hline & \multirow{2}{*}{ Rice } & Mixture & & Groundnuts \\
\cline { 2 - 4 } & & Potatoes & Pumpkins & 0.3 \\
\hline Production Amount (metric ton/acre) & 2 & 6.25 & 2.5 & 0.5 \\
Planting Cost (taka/acre) & 60,000 & 70,000 & 24,000 & 100,000 \\
Selling Price (taka/ metric ton) & 32,000 & 7,000 & 20,000 & - \\
Minimum Requirements (metric ton) & 0.5 & 0.25 & - & - \\
Total Land: 1 acre & & & & -000 \\
\hline
\end{tabular}

So, the linear programming problem for the above problem may be constructed as follows:

$$
\begin{array}{cc}
\text { Min } 60000 x_{1}+70000 x_{2}+24000 x_{3}+30000 x_{4}-32000 y_{1}-7000 y_{2}-20000 y_{3}-100000 y_{4}-60000 y_{5} \\
\text { Subject to, } x_{1}+x_{2}+x_{3}+x_{4} \leq 1, & y_{4} \leq 0.5 x_{4} \\
2 x_{1}-y_{1} \geq 0.5, & x_{1}, x_{2}, x_{3}, x_{4}, y_{1}, y_{2}, y_{3}, y_{4}, y_{5} \geq 0
\end{array}
$$

$$
\begin{gathered}
6.25 x_{2}-y_{2} \geq 0.25, \\
y_{5} \leq 2.5 x_{2}, \\
y_{3} \leq 0.3 x_{3},
\end{gathered}
$$

Now for the optimized values, we will solve the above linear programming problem by using LINDO solver (a software for solving linear programming problem): [8, 14]

LINDO solver file

\section{MIN 60000X1+70000X2+24000X3+30000X4-32000Y1-7000Y2-20000Y3-100000Y4-60000Y5}

$S T$

$\mathrm{Y} 5-2.5 \mathrm{X} 2<0$

$\mathrm{X} 1+\mathrm{X} 2+\mathrm{X} 3+\mathrm{X} 4<1$

$\mathrm{Y} 3-0.3 \mathrm{X} 3<0$

$2 \mathrm{X} 1-\mathrm{Y} 1>0.5$

$\mathrm{Y} 4-0.5 \mathrm{X} 4<0$

$6.25 \mathrm{X} 2-\mathrm{Y} 2>0.25$

END

Table 3. LINDO Solution for optimal prices.

\begin{tabular}{lll}
\hline OBJECTIVE FUNCTION VALUE & & \\
\hline 1) -76062.50 & & \\
\hline VARIABLE & VALUE & REDUCED COST \\
\hline X1 & 0.250000 & 0.000000 \\
X2 & 0.750000 & 0.000000 \\
X3 & 0.000000 & 141750.000000 \\
X4 & 0.000000 & 103750.000000 \\
Y1 & 0.000000 & 59875.000000 \\
Y2 & 4.437500 & 0.000000 \\
Y3 & 0.000000 & 0.000000 \\
Y4 & 0.000000 & 0.000000 \\
Y5 & 1.875000 & 0.000000 \\
\hline
\end{tabular}

Table 4. Optimal Solution.

\begin{tabular}{llllll}
\hline & \multirow{2}{*}{ Rice } & Mixture & Chilies & \multirow{2}{*}{ Groundnuts } \\
\cline { 3 - 5 } & & Potatoes & Pumpkins & - & - \\
\hline Land (acres) & 0.25 & 0.75 & & - & - \\
Production (metric ton) & 0.5 & 4.6875 & 1.875 & - & - \\
\hline
\end{tabular}




\begin{tabular}{llllll}
\hline & \multirow{2}{*}{ Rice } & Mixture & & Chilies & Groundnuts \\
\cline { 2 - 5 } & & Potatoes & Pumpkins & - & - \\
\hline Sell (metric ton) & - & 4.4375 & 1.875 & - & \\
Overall Profit: $76,062.50$ Taka & & & & \\
\hline
\end{tabular}

\subsection{Result Explanation}

The optimal solution describes that the farmer should spend his 0.25 acre land for cultivating rice which will fulfill his minimum requirements for his cattle feed and household consumes.
Raising potatoes and pumpkins is a mostly optimized category that gives him the best profit. He should devote his rest lands for raising potatoes and pumpkins. After satisfying the minimum requirements of potatoes, he can sell the rest of the products which will give him an amount of 76,062.50 Taka profit. And it is the most optimized profit for him.

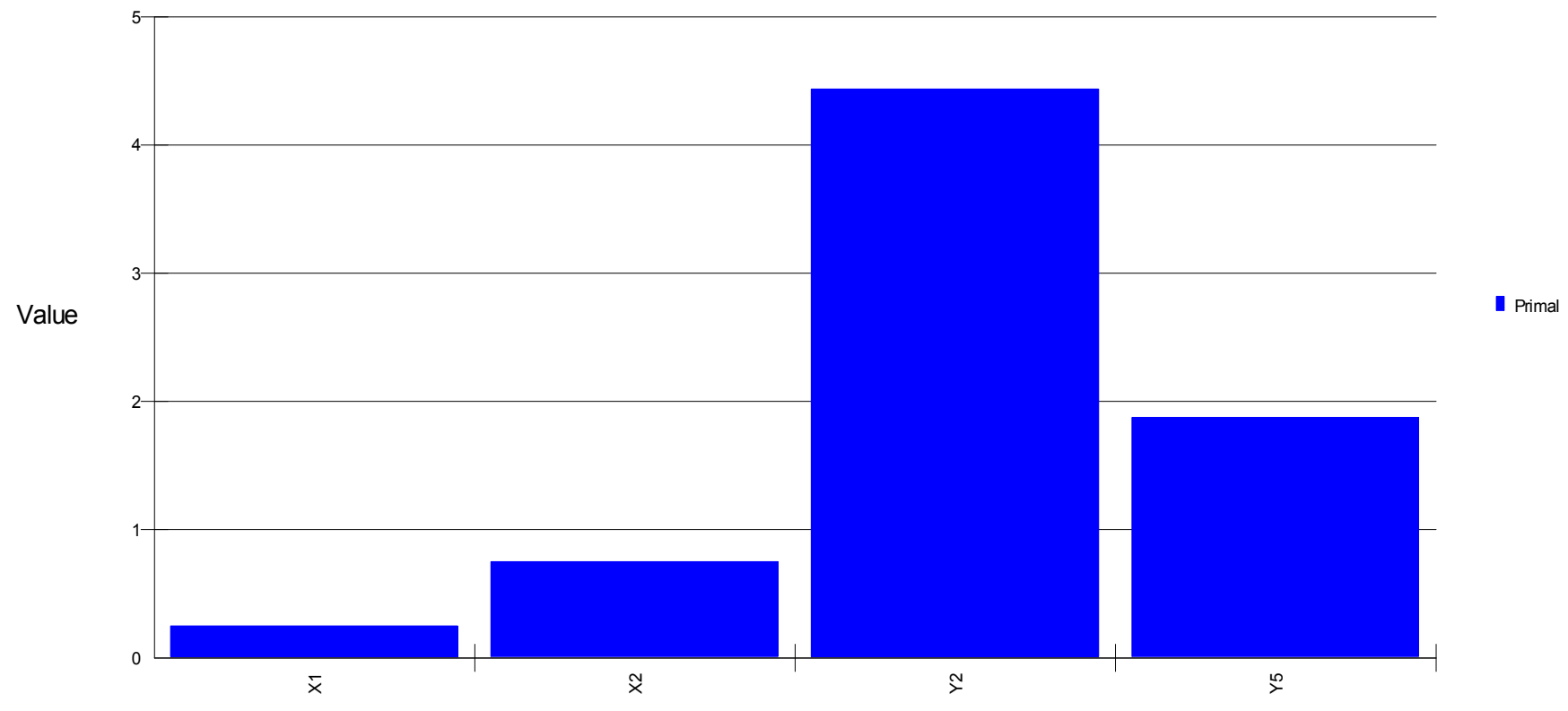

Figure 4. Optimized Values.

\subsection{Considering Stochastic Conditions}

Introducing Stochastic conditions means considering uncertainty. The above problem gives us the optimal condition with an average situation of planting cost, selling price, production amount, etc. In that case, all of these things depend on some uncertainty conditions. Considering Stochastic conditions means we may assume some fixed previous data or we can also take the average situation data.

In the above example, optimized values are calculated for the farmer with a neutral position about any type of risk. But if we think something about risk aversion, we have to follow this stochastic process. In this problem, the weather forecast could be a great option for cultivating crops. For example, if there is rain this winter (not considering time), rice production will be good but potatoes production will be hampered. And that will cost the farmer a lot since he chooses to cultivate potatoes according to the optimized values. In the same manner, if it is too hot this year rice production costs will be increased.

Actually, we calculated the above-optimized values by considering the average situation of weather based on past experience. We did not calculate the possible risks in this case.

Since we could not accurately predict the risks (like weather conditions, insect prevalence, water availability, market fluctuations, etc.) earlier like four months ago. So, for the risk aversion, we may consider the stochastic process and that could make a perfect decision that would be best in all circumstances.

For the above example, we may consider three situations: (considering $20 \%$ changes in profit).

Table 5. Considering Possible Situations.

\begin{tabular}{ll}
\hline Below average $(-20 \%)$ & 60,850 Taka \\
Average & $76,062.50$ Taka \\
Above Average $(+20 \%)$ & 91,275 Taka \\
\hline
\end{tabular}

Now for the construction of stochastic programming problem of the above model by using these three scenarios, we may consider some scenario index, $s=1,2,3$; which may create some new variables $y_{i s}(i=1,2,3,4,5)$. If the three scenarios have probabilities of $\frac{1}{4}$ for below average, $\frac{1}{2}$ for average and $\frac{1}{4}$ for above average, then the farmer's problem may be constructed as follows:

$$
\operatorname{Min} 60000 x_{1}+70000 x_{2}+24000 x_{3}+30000 x_{4}-\frac{1}{4}\left(32000 y_{11}+7000 y_{21}+20000 y_{31}+100000 y_{41}+60000 y_{51}\right)
$$




$$
\begin{array}{cc}
-\frac{1}{2}\left(32000 y_{12}+7000 y_{22}+20000 y_{32}+100000 y_{42}+60000 y_{52}\right)-\frac{1}{4}\left(32000 y_{13}+7000 y_{23}+20000 y_{33}+100000 y_{43}\right. \\
\left.+60000 y_{53}\right) \\
\text { Subject to, } x_{1}+x_{2}+x_{3}+x_{4} \leq 1 \\
1.6 x_{1}-y_{11} \geq 0.5 & y_{32} \leq 0.3 x_{3} \\
2 x_{1}-y_{12} \geq 0.5 & y_{33} \leq 0.36 x_{3} \\
2.4 x_{1}-y_{13} \geq 0.5 & y_{41} \leq 0.4 x_{4} \\
5 x_{2}-y_{21} \geq 0.25 & y_{42} \leq 0.5 x_{4} \\
6.25 x_{2}-y_{22} \geq 0.25 & y_{43} \leq 0.6 x_{4} \\
7.5 x_{2}-y_{23} \geq 0.25 & x_{1}, x_{2}, x_{3}, x_{4}, y_{11}, y_{12}, y_{13}, y_{21}, y_{22}, y_{23}, y_{31}, y_{32}, \\
y_{51} \leq 2 x_{2} & y_{33}, y_{41}, y_{42}, y_{43}, y_{51}, y_{52}, y_{53} \geq 0 \\
y_{52} \leq 2.5 x_{2} & \begin{array}{c}
\text { Such a model with stochastic decisions is known as the } \\
\text { extensive form of stochastic programming. } \\
\text { Now for the further optimized values, we will solve the } \\
\text { above linear programming problem by using LINDO solver } \\
\text { (a software for solving linear programming problem): [8, } 14] \\
\text { LINDO solver file }
\end{array}
\end{array}
$$

$$
\begin{gathered}
y_{32} \leq 0.3 x_{3} \\
y_{33} \leq 0.36 x_{3} \\
y_{41} \leq 0.4 x_{4} \\
y_{42} \leq 0.5 x_{4} \\
y_{43} \leq 0.6 x_{4}
\end{gathered}
$$$$
x_{1}, x_{2}, x_{3}, x_{4}, y_{11}, y_{12}, y_{13}, y_{21}, y_{22}, y_{23}, y_{31}, y_{32} \text {, }
$$$$
y_{33}, y_{41}, y_{42}, y_{43}, y_{51}, y_{52}, y_{53} \geq 0
$$

Such a model with stochastic decisions is known as the extensive form of stochastic programming.

Now for the further optimized values, we will solve the above linear programming problem by using LINDO solver (a software for solving linear programming problem): [8, 14] LINDO solver file

MIN 60000X1+70000X2+24000X3+30000X4-8000Y11-1750Y21-5000Y31-25000Y41-15000Y51-16000Y12-3500Y2210000Y32-50000Y42-30000Y52-8000Y13-1750Y23-5000Y33-25000Y43-15000Y53

$S T$

$$
\begin{gathered}
\mathrm{X} 1+\mathrm{X} 2+\mathrm{X} 3+\mathrm{X} 4<1 \\
1.6 \mathrm{X} 1-\mathrm{Y} 11>0.5 \\
2 \mathrm{X} 1-\mathrm{Y} 12>0.5 \\
2.4 \mathrm{X} 1-\mathrm{Y} 13>0.5 \\
5 \mathrm{X} 2-\mathrm{Y} 21>0.25 \\
6.25 \mathrm{X} 2-\mathrm{Y} 22>0.25 \\
7.5 \mathrm{X} 2-\mathrm{Y} 23>0.25 \\
\mathrm{Y} 51-2 \mathrm{X} 2<0
\end{gathered}
$$

$\mathrm{Y} 52-2.5 \mathrm{X} 2<0$

Y $53-3 \mathrm{X} 2<0$

$\mathrm{Y} 31-0.24 \mathrm{X} 3<0$

$\mathrm{Y} 32-0.3 \mathrm{X} 3<0$

$\mathrm{Y} 33-0.36 \mathrm{X} 3<0$

$\mathrm{Y} 41-0.4 \mathrm{X} 4<0$

$\mathrm{Y} 42-0.5 \mathrm{X} 4<0$

$\mathrm{Y} 43-0.6 \mathrm{X} 4<0$

Table 6. LINDO Solution Considering Stochastic Conditions.

\begin{tabular}{lll}
\hline OBJECTIVE FUNCTION VALUE & & \\
\hline 1) -68578.12 & & \\
\hline VARIABLE & VALUE & REDUCED COST \\
\hline X1 & 0.312500 & 0.000000 \\
X2 & 0.687500 & 0.000000 \\
X3 & 0.000000 & 141750.000000 \\
X4 & 0.000000 & 103750.000000 \\
Y11 & 0.000000 & 74843.750000 \\
Y21 & 3.187500 & 0.000000 \\
Y31 & 0.000000 & 0.000000 \\
Y41 & 0.000000 & 0.000000 \\
Y51 & 1.375000 & 0.000000 \\
Y12 & 0.125000 & 0.000000 \\
Y22 & 4.046875 & 0.000000 \\
Y32 & 0.000000 & 0.000000 \\
Y42 & 0.000000 & 0.000000 \\
Y52 & 1.718750 & 0.000000 \\
Y13 & 0.250000 & 0.000000 \\
\hline
\end{tabular}




\begin{tabular}{lll}
\hline OBJECTIVE FUNCTION VALUE & & \\
\hline 1) -68578.12 & & \\
\hline VARIABLE & VALUE & REDUCED COST \\
\hline Y23 & 4.906250 & 0.000000 \\
Y33 & 0.000000 & 0.000000 \\
Y43 & 0.000000 & 0.000000 \\
Y53 & 2.062500 & 0.000000 \\
\hline
\end{tabular}

Table 7. Optimal solution Based on Stochastic Model.

\begin{tabular}{|c|c|c|c|c|c|c|}
\hline \multirow[b]{2}{*}{ Situations } & \multirow{2}{*}{ Variables } & \multirow{2}{*}{ Rice } & \multicolumn{2}{|l|}{ Mixture } & \multirow{2}{*}{ Chilies } & \multirow{2}{*}{ Groundnuts } \\
\hline & & & Potatoes & Pumpkins & & \\
\hline First Stage Decision & Land (acres) & 0.3125 & 0.6875 & & - & - \\
\hline \multirow{2}{*}{$s=1$ Below Average } & Production (metric ton) & 0.5 & 3.4375 & 1.375 & - & - \\
\hline & Sell (metric ton) & - & 3.1875 & 1.375 & - & - \\
\hline \multirow{2}{*}{$s=2$ Average } & Production (metric ton) & 0.625 & 4.296875 & 1.71875 & - & - \\
\hline & Sell (metric ton) & 0.125 & 4.046875 & 1.71875 & - & - \\
\hline \multirow{2}{*}{$s=3$ Above Average } & Production (metric ton) & 0.75 & 5.15625 & 2.0625 & - & - \\
\hline & Sell (metric ton) & 0.25 & 4.90625 & 2.0625 & - & - \\
\hline \multicolumn{7}{|c|}{ Overall Profit: 68578.12 Taka } \\
\hline
\end{tabular}

\subsection{Result Explanation Based on Stochastic}

Here the optimized amount of lands gives the planting areas, which has to be determined before the weather forecast and crop production. This is the first stage decision.
The other descriptions are productions, sales in three scenarios. They are the second stage decisions.

The last line gives us the overall profit which is $7,484.38$ Taka less than the first optimized profit. Actually, it is the risk aversion cost.

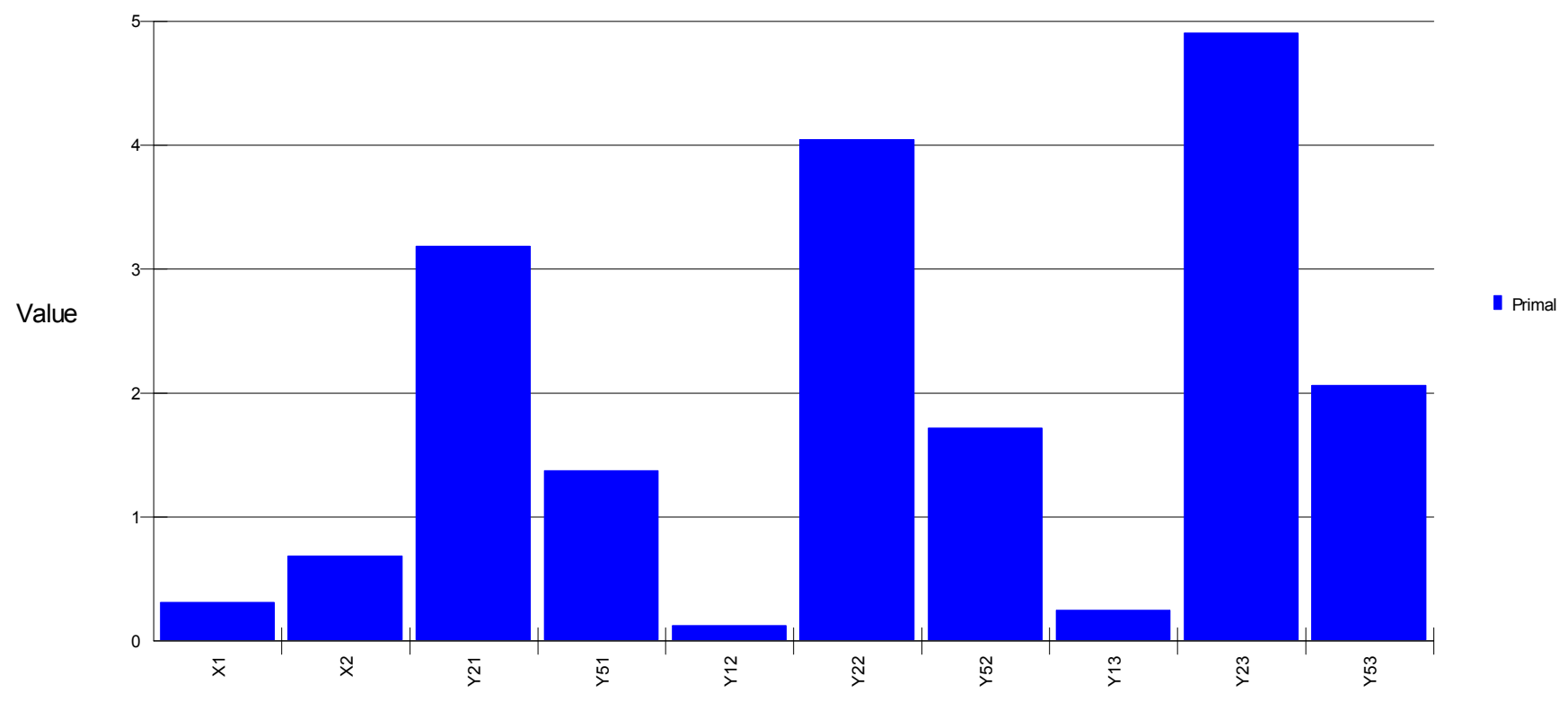

Figure 5. Optimized Values based on Stochastic Model.

\section{Applications of Stochastic Programming}

Randomness is everywhere and that says that stochastic programming has a variety of applications in every sector of life, society, business, etc. Based on the works on Stochastic Programming until now, we may classify the applications of Stochastic Programming into the following sections: [19]

i. Production, Supply Chain and Scheduling

a) Managing fleets of trailers, containers, boxcars, locomotives, business jets, etc.

b) Problems with uncertain parameters like product price and demand, raw materials supply cost, production cost, operation execution durations, resource availability, etc. are the root approaches to Benders Decomposition, Lagrangian Decomposition.

c) Supply Chain optimization in the meat industry, developed for Norwegian meat.

d) Melt control model with uncertain sources.

e) Network resource utilization in the presence of multiclass demand uncertainty.

ii. Gaming

a) High-performance yacht racing with uncertain route optimization.

b) Stochastic approximation of momentum and Nash 
Play.

iii. Environment and Pollution

a) Lake eutrophication (a process by which pollution over-rich in organic and mineral nutrients) management.

b) Design of hedging experiments in stochastic environments where growth trajectories, climate sensitivities, and damage estimates are uncertain.

c) Controlling a groundwater contaminant plume.

d) Catastrophic risk management via flood and seismic risk case studies.

iv. IFinance

a) Refinancing mortgages with noncontractual maturity under liquidity restrictions in the market.

b) Structuring index funds.

c) Decentralized Risk management for Global $\mathrm{P} / \mathrm{C}$ Insurance Companies.

d) Asset allocation (invest) involving stocks, bonds, and cash wit wealth goals.

e) Scenario-based risk management tool.

f) Price protection strategies.

g) Optimization of hedge funds.

v. Telecom and Electricity

a) A Short-term unit commitment of a hydrothermal power system under uncertainty to load, inflow to reservoirs, and prices for fuel and delivery contracts.

b) Valuation of electricity generation capacity incorporating operational constraints such as start-up cost, ramp-up cost, and operating level-dependent heat rate.

c) Telecommunication design problems with stochastic programming paradigm.

There are more works on stochastic programming applications. These are some samples.

\section{Concluding Remarks}

In this paper, we presented how risk arises in optimization techniques with stochastic programming approaches, from the starting of considering uncertainty with a stochastic process. We also described a model to make harvesting decisions and described the points that arise in stochastic programming with the model. According to the model, A farmer has to lose some profit by considering the risks in harvesting. But it could be more beneficial in the long run since it gives insurance for the farmer for any kind of risk. This also opens a variety of sections for modeling different systems or phenomena with stochasticity.

\section{References}

[1] Birge, John R., and Francois Louveaux. Introduction to stochastic programming. Springer Science \& Business Media, 2011.

[2] Kall, Peter, and Janos Mayer. Stochastic linear programming. Vol. 7. Berlin: Springer-Verlag, 1976.
[3] Wallace, Stein W., and William T. Ziemba, eds. Applications of stochastic programming. Society for Industrial and Applied Mathematics, 2005.

[4] Ahmed, Hashnayne. "Graph Routing Problem Using Euler's Theorem and Its Applications." (2019).

[5] Dantzig, George B. "Linear programming under uncertainty." Stochastic programming. Springer, New York, NY, 2010. 111.

[6] Sen, Suvrajeet, and Julia L. Higle. "An introductory tutorial on stochastic linear programming models." Interfaces 29.2 (1999): 33-61.

[7] Tintner, Gerhard. "A note on stochastic linear programming." Econometrica: Journal of the Econometric Society (1960): 490-495.

[8] Molla, Md Hasib Uddin, and M. Babul Hasan. "Art of Formulating LPs and IPs from Real Life Problems." Dhaka University Journal of Science 61.2 (2013): 185-191.

[9] Spall, James C. "Simultaneous perturbation stochastic approximation." Introduction to stochastic search and optimization: Estimation, simulation, and control (2003): 176207.

[10] Doob, Joseph Leo. Stochastic processes. Vol. 101. Wiley: New York, 1953.

[11] Kumar, Panqanamala Ramana, and Pravin Varaiya. Stochastic systems: Estimation, identification, and adaptive control. Vol. 75. SIAM, 2015.

[12] Ghaffari-Hadigheh, Alireza, and Zeinab Zarea. "One-Stage Uncertain Linear optimization." Journal of Hyperstructures 7.1 (2019).

[13] King, Alan J., and Stein W. Wallace. Modeling with stochastic programming. Springer Science \& Business Media, 2012.

[14] Inc, Lindo Systems. "LINDO User's Manuals." LINDO Systems, Inc (2003).

[15] Wu, Hao-Hsiang, and Simge Küçükyavuz. "A two-stage stochastic programming approach for influence maximization in social networks." Computational Optimization and Applications 69.3 (2018): 563-595.

[16] Ameri, Mahmoud, et al. "A Two-Stage Stochastic Model for Maintenance and Rehabilitation Planning of Pavements." Mathematical Problems in Engineering, 2019.

[17] $\mathrm{Xu}, \mathrm{Ye}$, et al. "Stochastic optimization model for water allocation on a watershed scale considering wetland's ecological water requirement." Ecological Indicators 92 (2018): 330-341.

[18] Bertsimas, Dimitris, and Nataly Youssef. "Stochastic optimization in supply chain networks: averaging robust solutions." Optimization Letters (2019): 1-17.

[19] Gassmann, Horand I., et al. "Introduction to stochastic programming applications." Applications of stochastic programming. Society for Industrial and Applied Mathematics, 2005. 179-184.

[20] Sultan, Alan. Linear programming: An introduction with applications. Elsevier, 2014. 
[21] Ahmed, Hashnayne. "Formulation of Two-Stage Stochastic Programming with Fixed Recourse." Britain International of Exact Sciences (BIoEx) Journal 1.1 (2019): 18-21.
[22] Ahmed, Hashnayne. "A Proposed Linear Programming Based Algorithm to solve Arc Routing Problems." International Journal of Mathematical Sciences and Computing 6.1 (2020). 